\title{
The visceral fat area to leg muscle mass ratio is significantly associated with the risk of hyperuricemia among women: a cross- sectional study
}

Xiao-He Wang ${ }^{1}$, Wei-Ran Jiang ${ }^{3}$, Min-Ying Zhang ${ }^{2}$, Ying-Xin Shi ${ }^{1}$, Yun-Ping Ji ${ }^{1}$, Chun-Jun Li ${ }^{1 *}$ (D and Jing-Na Lin ${ }^{1 *}$

\begin{abstract}
Background: A significant positive association was found in previous studies among obesity, visceral fat accumulation, and hyperuricemia. The purpose of this study was to explore the association between the ratio of visceral fat area to leg muscle mass (VFA-to-LMM) and hyperuricemia, and verify the role of gender differences in the association.

Methods: A total of 3393 (43.3\% are men) participants from Tianjin Union Medical Center-Health Management Center were recruited for this cross-sectional study. The VFA-to-LMM ratio was used as the independent variable. Hyperuricemia, a serum uric acid level $\geq 416 \mu \mathrm{mol} / \mathrm{L}$ in men and in menopausal women and $\geq 357 \mu \mathrm{mol} / \mathrm{L}$ in premenopausal women, was used as the dependent variable. Multiple logistic regression analysis was used to estimate the odds ratio and the 95\% confidence interval between the VFA-to-LMM ratio and hyperuricemia.

Results: The overall prevalence of hyperuricemia was 14.8\% (8.9\% in women, and $22.5 \%$ in men). After adjustment by age, smoking status (for males), menopause status (for females), drinking status, exercise frequency, blood pressure, alanine aminotransferase, fasting plasma glucose, triglycerides, low-density lipoprotein cholesterol, highdensity lipoprotein cholesterol, creatinine, and history of diseases, a strong positive association was found between the VFA-to-LMM ratio and hyperuricemia in both men (4th vs. 1st quartile 1.60, 95\% Cl: 1.03-2.49) and women (4th vs. 1st quartile $5.22,95 \% \mathrm{Cl}$ : 2.44-12.56). After additional adjustment by BMl, there was still a significant positive association in women (4th vs. 1st quartile $2.57,95 \% \mathrm{Cl}$ : 1.06-6.77). The results of subgroup analysis showed that premenopausal women (4th vs. 1st quartile OR: 3.61) have a higher risk of hyperuricemia than postmenopausal women (4th vs. 1st quartile OR: 1.94) with the increase of the VFA-to-LMM ratio. Besides, the interaction analysis results showed the highest risk of hyperuricemia when VFA and LMM were both in the highest quantile (OR: 11.50; 95\% Cl: 4.86-31.98).

\footnotetext{
*Correspondence: li_chunjun@126.com; 13207628978@163.com

1 Department of Endocrinology, Health Management Center, Tianjin Union Medical Center, Nankai University Affiliated Hospital, 190 of Jieyuan Road, Hongqiao District, Tianjin 300121, China

Full list of author information is available at the end of the article
}

(c) The Author(s). 2021 Open Access This article is licensed under a Creative Commons Attribution 4.0 International License, which permits use, sharing, adaptation, distribution and reproduction in any medium or format, as long as you give appropriate credit to the original author(s) and the source, provide a link to the Creative Commons licence, and indicate if changes were made. The images or other third party material in this article are included in the article's Creative Commons licence, unless indicated otherwise in a credit line to the material. If material is not included in the article's Creative Commons licence and your intended use is not permitted by statutory regulation or exceeds the permitted use, you will need to obtain permission directly from the copyright holder. To view a copy of this licence, visit http://creativecommons.org/licenses/by/4.0/ The Creative Commons Public Domain Dedication waiver (http://creativecommons.org/publicdomain/zero/1.0/) applies to the data made available in this article, unless otherwise stated in a credit line to the data. 
(Continued from previous page)

Conclusion: The VFA-to-LMM ratio was positively associated with the risk of hyperuricemia in women after adjustment by confounders. Pre-menopausal women have a higher risk of hyperuricemia than postmenopausal women with the increase of the VFA-to-LMM ratio. In addition, the highest risk of hyperuricemia was demonstrated when both VFA and LMM were at the highest quartile.

Keywords: Body composition, Visceral fat area, Skeletal muscle mass, Metabolic diseases, Hyperuricemia, Chinese adults

\section{Introduction}

According to the National Health and Nutrition Examination Survey [1], the prevalence rates of hyperuricemia were $20.2 \%$ for men and $20.0 \%$ for women during $2007-$ 2016, and the rates did not show any decline in the decade. Compared with the high prevalence of hyperuricemia in the USA, although China has a relatively low prevalence of hyperuricemia $(19.4 \%$ in men and $7.9 \%$ in women) [2], it is still rising according to the latest data [3]. Hyperuricemia, a metabolic disease, is closely related to the inflammatory response and the disorder of glucose and lipid metabolism [4], which plays an important role in the formation of metabolic syndrome [5]. In addition, hyperuricemia, inflammation, and oxidative stress can accelerate the process of endothelial dysfunction, which is the pathophysiological basis of metabolic syndrome [6]. Moreover, hyperuricemia has also been shown to be significantly associated with lifestyle-related chronic diseases, such as hypertension, diabetes [7], non-alcoholic fatty liver disease (NAFLD) [8], and cancer. In addition, a meta-analysis based on cohort studies also confirmed that hyperuricemia was associated with high cancer incidence and mortality [9].

Considering the serious health outcomes caused by hyperuricemia, plenty of researchers have started to study its risk factors. A large population-based study confirmed that four modifiable risk factors, including the body mass index (BMI), alcohol use, diuretic use, and Dietary Approaches to Stop Hypertension diet, are independently associated with the development of hyperuricemia [10]. Among these modifiable risk factors, BMI is a comprehensive indicator that could indirectly reflect the overall condition of diet, physical activity, and metabolism. There is a complex correlation between obesity and hyperuricemia. The former can cause hyperuricemia by increasing uric acid synthesis and inhibiting its excretion, and (in turn) increased uric acid levels can promote the development of obesity by accelerating body fat accumulation (especially visceral fat) [11]. However, BMI fails to reflect the metabolic differences between men and women, such as fat distribution and skeletal muscle mass (SMM). In fact, studies have proved that there was a positive association between visceral fat and hyperuricemia [12-14], and a significant inverse association between serum uric acid (SUA) levels and SMM $[15,16]$.
Furthermore, studies have shown that leg composition was associated with some metabolic diseases [17, 18]. The thigh circumference which indirectly reflects leg composition has also been confirmed to be associated with various metabolic diseases, such as insulin resistance (IR), carotid atherosclerosis [19], and type-2 diabetes [20]. One explanation is that decreased SMM and fat mass in the lower limbs may be associated with disorders of glucose and lipid metabolism [21], which is closely related to hyperuricemia $[22,23]$.

Based on the previous studies, it can be speculated that the visceral fat area (VFA) and the leg muscle mass (LMM) seemed to have played a key role in the pathogenesis of hyperuricemia, and the combination of the two indicators would be a potential predictor of hyperuricemia. Therefore, a cross-sectional study was designed in this paper to explore the association between the VFA-to-LMM ratio and hyperuricemia, and to verify the role of gender differences in the association, so as to find a clinically feasible and gender-sensitive predictor for the risk of hyperuricemia.

\section{Methods}

\section{Participants}

The target population for the study was those who visited Tianjin Union Medical Center-Health Management Center for an annual physical examination from September 2019 to December 2019.

Before body composition examination, participants were interviewed and those who had recently taken specific drugs (diuretics, hypoglycemic agents, aspirin, vitamin $C$, etc.) were excluded because these drugs might affect uric acid metabolism. A total of 4084 eligible adults were enrolled in the study, among which 602 were excluded because they did not provide complete information, including physical examinations, biochemical analysis, questionnaires, and body composition, 9 were excluded because they had a history of cancer, and 52 were excluded because they were above 85 years old, as the association between body composition and hyperuricemia due to stability of physiological indicators or serious declination of health status might be affected. Besides, the participants $(n=28)$ who have extreme values in the measurement indicators were also excluded. Finally, 3393 ( $83 \%$ of those eligible) subjects had 
valid body composition data and covariate information required for the cross-sectional study (Fig. 1).

\section{Measurement of body composition}

Body composition was measured by multielectrode bioelectrical impedance analyzer (Inbody 770, Biospace Inc., Korea). According to the manufacturer's guidelines, subjects were required to fast overnight and wipe the bottom of their feet with clean water before taking measurements. They were instructed to stand barefoot on the base components with light clothing and grasp the handles of the instrument to ensure full contact with a total of 8 electrodes. Then they were asked to slightly abduct their arms and maintain the posture during the assessment. The entire measurement process took about 3-4 $\mathrm{min}$, and the data were automatically saved in the computer after the measurement was completed. Based on the purpose of this study, the VFA-to-LMM ratio was selected as the independent variable.

\section{Covariables}

Weight and height were measured by an automatic height and weight instrument (DST-600, DONGHUAYUAN, China), and participants were required to stay barefooted with light clothing during the measurement. BMI was calculated as weight in kilograms divided by the square of height in meters. All measurements are carried out in strict accordance with the national standards.

Blood pressure was measured using an automatic electronic blood pressure monitor (AC-05C, Ling Qian, China) after a 10-min rest. Each person was measured three times and the average value was taken. Hypertension is defined as systolic blood pressure $(\mathrm{SBP}) \geq 140 \mathrm{mmHg}$ and/or diastolic

Participants enrolled in the cross-sectiona study during Sep 2019 to Dec 2019 (n=4084)

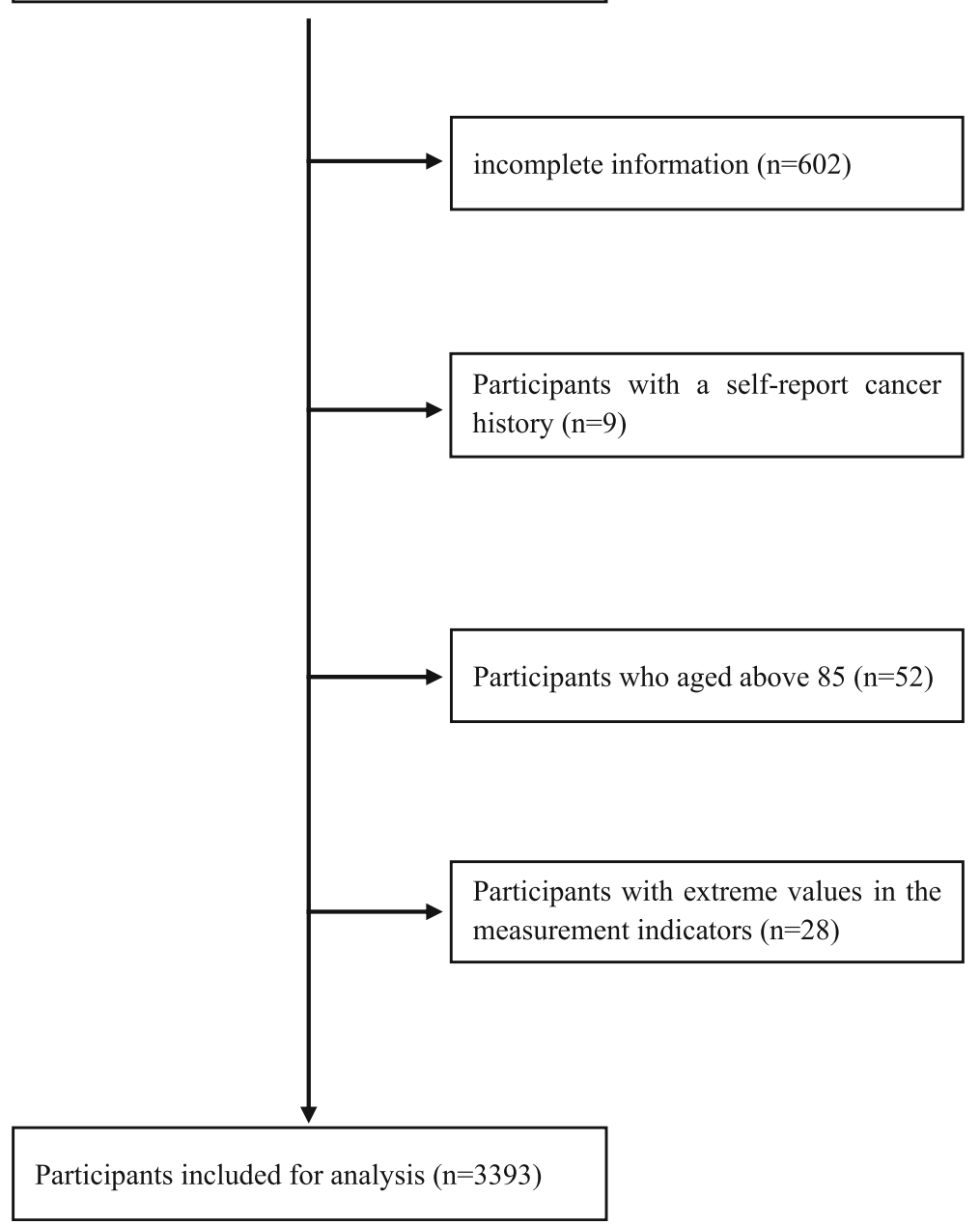

Fig. 1 Flowchart showing the selection of the studied population 
blood pressure $(\mathrm{DBP}) \geq 90 \mathrm{mmHg}$ or with a history of hypertension. Ultrasound liver examination was performed by an experienced ultrasound physician using an ultrasound diagnosis system (Phoenix, Philips and Neusoft Medical Systems Co., Ltd., China). The diagnosis of NAFLD was based on imaging diagnostic criteria [24]: (1) the nearfield echo of the liver is diffusely enhanced (bright liver), and liver echogenicity is greater than that of kidney or spleen; (2) vascular blurring; and (3) deep attenuation of ultrasound signal. Blood biochemical analysis was performed using an automatic biochemical analyzer (TBA120FR, Toshiba, Japan), and participants were required to fast overnight (only allowed to drink water), and venous blood was collected in a fasting state. The main indicators of blood biochemical analysis include alanine aminotransferase (ALT), fasting plasma glucose (FPG), total cholesterol (TC), triglycerides (TG), low-density lipoprotein (LDL-C), high-density lipoprotein (HDL-C) cholesterol, and serum uric acid. Diabetes is defined as FPG $\geq 7.0$ or with a history of diabetes. Dyslipidemia is defined as TC $\geq 6.2 \mathrm{mmol} / \mathrm{L}$ or $\mathrm{TG} \geq 2.3 \mathrm{mmol} / \mathrm{L}$ or $\mathrm{LDL}-\mathrm{C} \geq 4.1 \mathrm{mmol} / \mathrm{L}$ or $\mathrm{HDL}-\mathrm{C}<1.0$ $\mathrm{mmol} / \mathrm{L}$ according to the Chinese Guidelines for the Management of Dyslipidemia in Adults (2016) [25]. Hyperuricemia is defined as a serum uric acid level $\geq 416 \mu \mathrm{mol} / \mathrm{L}$ in men and menopausal women, and $\geq 357 \mu \mathrm{mol} / \mathrm{L}$ in premenopausal women.

The socio-demographic variables, such as gender, age, and menopause status, were collected through questionnaires. Information on lifestyles, including exercise frequency ("never," "occasional," and "regularly"), smoking status ("smoker," "Ex-smoker," and "Non-smoker"), drinking status ("drinker," and "ex-drinker," and "non-drinker"), and sedentary status ( $>6 \mathrm{~h} /$ day), was also obtained in questionnaires. As for the history of diseases (including diabetes, hypertension, coronary heart disease, and cancer), participants were required to answer a "yes" or "no".

\section{Statistical analysis}

The characteristics of participants were presented as the means \pm standard deviation or the median (interquartile range) for continuous variables and percentages for categorical variables. Statistical differences between groups were examined through analysis of variance for continuous variables and Chi-square test for categorical variables. Multiple logistic regression analysis was used to estimate the odds ratios (ORs) and 95\% confidence intervals (CIs) between the VFA-to-LMM ratio and hyperuricemia. For further analysis, we fitted four models and performed a quartile conversion of the independent variable. Model 1 was adjusted by age and model 2 was adjusted by age, smoking status (for males), menopause status (for females), drinking status, exercise frequency and history of diseases. Model 3 was additionally adjusted by SBP, DBP, ALT, FPG, TC, TG, LDL-C, HDL-
C, and creatinine. Model 4 was additionally adjusted by BMI based on model 3. All statistical analyses were performed with SAS 9.4 for Windows (SAS Institute, Cary, NC, USA). $P$ values were two-tailed, and the differences were considered to be significant when $P<0.05$.

\section{Results \\ Characteristics of participants}

The characteristics of participants by gender and hyperuricemia status are listed in Table 1 . The overall prevalence of hyperuricemia was $14.8 \%$ (22.5\% in men and $8.9 \%$ in women), and the average age of participants was 45.9 years. Analysis of the differences between the hyperuricemia group and non-hyperuricemia group indicated that there were significant differences in almost all indicators in both men and women, except for smoking, drinking, exercise, and history of diseases. Indicators, such as SBP, FPG, hypertension, and diabetes, were observed to have significant differences only in women, and indicators, such as LMM and regular exercise, were discovered to have significant differences only in men. The differences in the VFA-to-LMM ratio between hyperuricemia and non-hyperuricemia were shown in Fig. 2. According to this figure, there were significant differences in the VFA-to-LMM ratios not only between hyperuricemia and non-hyperuricemia but also between men and women $(P<.0001)$.

Furthermore, the subjects were divided into four groups based on the quartiles of the VFA-to-LMM ratio, and the linear trend of each indicator (Table 2) was analyzed. As the VFA-to-LMM ratio increased, most of the indicators (BMI, FPG, TG, LDL-C, SUA) witnessed a significant linear upward trend in both men and women, and only HDL-C was found to have a downward trend.

\section{VFA-to-LMM ratio and risk of hyperuricemia}

The association between the VFA-to-LMM ratio and hyperuricemia was explored through multiple logistic regression models, and the results were exhibited in Table 3. After adjustment by age in model 1, a strong positive association was observed between the VFA-toLMM ratio and hyperuricemia in both men (4th vs. 1st quartile OR: 2.73 ; 95\% CI: $1.89-3.95$ ) and women (4th vs. 1st quartile OR: 8.25 ; $95 \%$ CI: $4.05-19.14)$, and significant linear trends were discovered in both men $(P<$ $0.0001)$ and women $(P<0.0001)$. After additional adjustment by smoking status (for men), menopause status (for women), drinking status, exercise frequency, SBP, DBP, ALT, FPG, TC, TG, LDL-C, HDL-C, creatinine, and history of diseases in model 3 , there were still significant positive association between the VFA-to-LMM ratio and hyperuricemia in both men (4th vs. 1st quartile OR: 1.60; 95\% CI: $1.03-2.49$ ) and women (4th vs. 1st quartile OR: $5.22 ; 95 \%$ CI: 2.44-12.56). To further 
Table 1 Characteristics of the study population according to sex and hyperuricemia status

\begin{tabular}{|c|c|c|c|c|c|c|}
\hline \multirow[t]{2}{*}{ Characteristics } & \multicolumn{2}{|l|}{ Men $(n=1469)$} & \multirow[t]{2}{*}{$P^{\mathrm{a}}$} & \multicolumn{2}{|l|}{ Women $(n=1924)$} & \multirow[t]{2}{*}{$P$} \\
\hline & $\begin{array}{l}\text { Non-hyperuricemia } \\
(n=1139)\end{array}$ & $\begin{array}{l}\text { Hyperuricemia } \\
(n=330)\end{array}$ & & $\begin{array}{l}\text { Non-hyperuricemia } \\
(n=1753)\end{array}$ & $\begin{array}{l}\text { Hyperuricemia } \\
(n=171)\end{array}$ & \\
\hline Age, years & $47.9 \pm 15.7$ & $44.4 \pm 14.6$ & 0.0002 & $44.0 \pm 14.5$ & $54.4 \pm 16.1$ & $<.0001$ \\
\hline $\mathrm{BMI}, \mathrm{kg} / \mathrm{m}^{2}$ & $25.4 \pm 3.2$ & $27.0 \pm 3.5$ & $<.0001$ & $22.8 \pm 3.2$ & $26.0 \pm 3.9$ & $<.0001$ \\
\hline $\mathrm{SBP}, \mathrm{mmHg}$ & $127.0 \pm 16.8$ & $127.8 \pm 15.4$ & 0.4377 & $117.3 \pm 18.1$ & $129.8 \pm 19.3$ & $<.0001$ \\
\hline $\mathrm{DBP}, \mathrm{mmHg}$ & $80.2 \pm 10.0$ & $83.4 \pm 10.0$ & $<.0001$ & $75.4 \pm 9.7$ & $79.1 \pm 11.5$ & $<.0001$ \\
\hline VFA, $\mathrm{cm}^{2}$ & $90.7 \pm 32.8$ & $104.9 \pm 36.6$ & $<.0001$ & $95.1 \pm 35.4$ & $129.1 \pm 41.0$ & $<.0001$ \\
\hline LMM, kg & $17.4 \pm 2.2$ & $18.0 \pm 2.2$ & $<.0001$ & $12.3 \pm 1.6$ & $12.5 \pm 1.8$ & 0.1409 \\
\hline ALT, units/L & $21.6(15.3,31.6)^{b}$ & $27.3(18.8,45.5)$ & $<.0001$ & $13.8(10.0,19.6)$ & $18.3(13.6,26.0)$ & $<.0001$ \\
\hline $\mathrm{FPG}, \mathrm{mmol} / \mathrm{L}$ & $5.3(4.9,5.9)$ & $5.2(5.0,5.7)$ & 0.3329 & $5.1(4.8,5.4)$ & $5.6(5.1,6.5)$ & $<.0001$ \\
\hline $\mathrm{TC}, \mathrm{mmol} / \mathrm{L}$ & $5.0 \pm 0.9$ & $5.2 \pm 0.9$ & $<.0001$ & $5.1 \pm 1.0$ & $5.8 \pm 1.2$ & $<.0001$ \\
\hline $\mathrm{TG}, \mathrm{mmol} / \mathrm{L}$ & $1.3(0.9,1.8)$ & $1.7(1.2,2.3)$ & $<.0001$ & $1.0(0.7,1.4)$ & $1.6(1.1,2.2)$ & $<.0001$ \\
\hline LDL-C, mmol/L & $2.6 \pm 0.5$ & $2.8 \pm 0.5$ & $<.0001$ & $2.7 \pm 0.6$ & $3.1 \pm 0.7$ & $<.0001$ \\
\hline $\mathrm{HDL}-\mathrm{C}, \mathrm{mmol} / \mathrm{L}$ & $1.3 \pm 0.2$ & $1.3 \pm 0.2$ & $<.0001$ & $1.6 \pm 0.3$ & $1.5 \pm 0.3$ & $<.0001$ \\
\hline SUA, $\mu \mathrm{mol} / \mathrm{L}$ & $336.0 \pm 50.3$ & $468.7 \pm 47.1$ & $<.0001$ & $260.4 \pm 45.8$ & $395.3 \pm 38.0$ & $<.0001$ \\
\hline Creatinine, $\mu \mathrm{mol} / \mathrm{L}$ & $74.7 \pm 10.0$ & $79.8 \pm 11.7$ & $<.0001$ & $55.6 \pm 7.7$ & $61.5 \pm 11.6$ & $<.0001$ \\
\hline Smoking, $n$ (\%) & $247(21.7)$ & $69(20.9)$ & 0.7624 & $13(0.7)$ & $0(0.0)$ & 0.6208 \\
\hline Drinking, $n(\%)$ & $459(40.3)$ & $129(39.1)$ & 0.6934 & $107(6.1)$ & $5(2.9)$ & 0.1275 \\
\hline \multicolumn{7}{|l|}{ Exercise, $n$ (\%) } \\
\hline Never & $285(25.0)$ & $95(28.8)$ & 0.1689 & $461(26.3)$ & $50(29.2)$ & 0.4057 \\
\hline Occasional & $380(33.4)$ & $124(37.6)$ & 0.1557 & $733(41.8)$ & $63(36.8)$ & 0.2076 \\
\hline Regular & $474(41.6)$ & 111 (33.6) & 0.0091 & $559(31.9)$ & $58(33.9)$ & 0.5872 \\
\hline Hypertension, n (\%) & $355(31.2)$ & $114(34.6)$ & 0.2465 & $288(16.4)$ & $71(41.5)$ & $<.0001$ \\
\hline Diabetes, n (\%) & $124(10.9)$ & $28(8.5)$ & 0.2071 & $69(3.9)$ & $26(15.2)$ & $<.0001$ \\
\hline Dyslipidemia, n (\%) & $258(22.7)$ & $131(39.7)$ & $<.0001$ & $316(18.0)$ & $76(44.4)$ & $<.0001$ \\
\hline NAFLD, n (\%) & $473(41.5)$ & $211(63.9)$ & $<.0001$ & $405(23.1)$ & $116(67.8)$ & $<.0001$ \\
\hline Obesity, n (\%) & $232(20.4)$ & $122(37.0)$ & $<.0001$ & $123(7.0)$ & $36(21.1)$ & $<.0001$ \\
\hline \multicolumn{7}{|c|}{ History of diseases, $n$ (\%) } \\
\hline Hypertension & $367(32.2)$ & $111(33.6)$ & 0.6290 & $317(18.1)$ & 75 (43.9) & $<.0001$ \\
\hline Diabetes & $116(10.2)$ & $23(7.0)$ & 0.0789 & $84(4.8)$ & $25(14.6)$ & $<.0001$ \\
\hline $\mathrm{CHD}$ & $68(6.0)$ & $18(5.5)$ & 0.7254 & $53(3.0)$ & 19 (11.1) & $<.0001$ \\
\hline
\end{tabular}

$B M I$ body mass index, SBP systolic blood pressure, DBP diastolic blood pressure, VFA visceral fat area, $L M M$ leg muscle mass, $A L T$ alanine aminotransferase, FPG fasting plasma glucose, TC total cholesterol, TG triglycerides, $L D L-C$ low-density lipoprotein cholesterol, $H D L-C$ high-density lipoprotein cholesterol, SUA serum uric acid, NAFLD non-alcoholic fatty liver diseases, CHD coronary heart disease

Continuous variables are expressed as means \pm standard deviation (SD) and categorical variables are expressed as percentages unless otherwise indicated

${ }^{a}$ Analysis of variance or chi-square test where appropriate

${ }^{\mathrm{b}}$ Data are expressed as medians (interquartile range)

analyze whether the association was independent of general obesity, BMI was added as an adjustment factor in model 3. According to the results, there was still a significant positive association between the VFA-to-LMM ratio and hyperuricemia in women (4th vs. 1st quartile OR: 2.57 ; 95\% CI: 1.06-6.77), while no association was observed in men (4th vs. 1st quartile OR: 0.97; 95\% CI: $0.55-1.70)$.

After that, a comparison was made on the prevalence of hyperuricemia and the odds ratios of hyperuricemia between men and women according to the quartiles of the VFA-to-LMM ratio. As shown in Fig. 3, the prevalence of hyperuricemia in women was significantly lower than that in men, but the risk of hyperuricemia in women was much higher than that in men as the VFAto-LMM ratio increased.

\section{Subgroup analysis in women according to menopausal status}

As shown in Table 4, the association between the VFAto-LMM ratio and hyperuricemia in women with different menopausal status was demonstrated. According to 


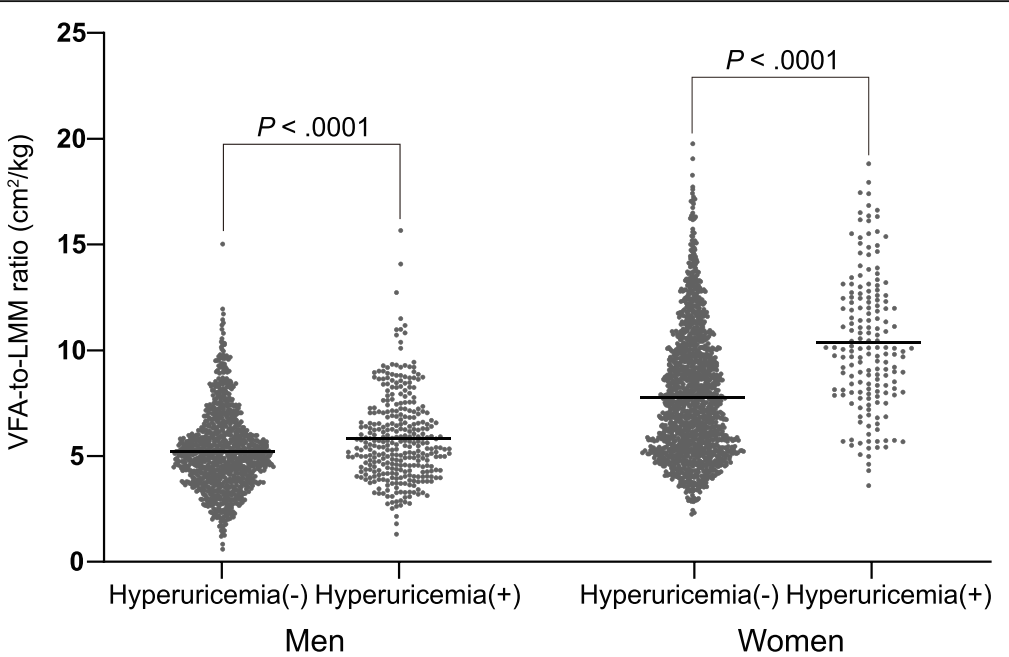

Fig. 2 The differences in the VFA-to-LMM ratio between hyperuricemia and non-hyperuricemia among men and women. VFA visceral fat area, LMM leg muscle mass

the results, pre-menopausal women had a significantly higher risk of hyperuricemia than post-menopausal women. However, the association between the two groups was no longer significant after adjustment by BMI in model 4. It is worth noting that although the association was attenuated, the OR values of premenopausal women (4th vs. 1st quartile OR: 3.61 ) was still higher than that of postmenopausal women (4th vs. 1st quartile OR: 1.94), and the $P$ value for trends was closer to $0.05(P=0.1503)$.

\section{The interaction of VFA and LMM on the risk of hyperuricemia}

In the further analysis, we divided the subjects into 16 groups according to the quartiles of VFA and LMM (the lowest quartile of VFA and the lowest quartile of LMM

Table 2 Characteristics of subjects according to quartiles of VFA-to-LMM ratio

\begin{tabular}{|c|c|c|c|c|c|}
\hline \multirow[t]{2}{*}{ Variables of interest } & \multicolumn{4}{|c|}{ Quartiles of VFA-to-LMM ratio } & \multirow[t]{2}{*}{$P$ for Trend ${ }^{a}$} \\
\hline & Q1 & Q2 & Q3 & Q4 & \\
\hline \multicolumn{6}{|l|}{ Men } \\
\hline No. of subjects & 367 & 365 & 369 & 368 & \\
\hline $\mathrm{BMI}, \mathrm{kg} / \mathrm{m}^{2}$ & $22.6 \pm 2.4$ & $25.2 \pm 2.2$ & $26.1 \pm 2.1$ & $29.0 \pm 3.1$ & $<.0001$ \\
\hline $\mathrm{FPG}, \mathrm{mmol} / \mathrm{L}$ & $5.1(4.8,5.5)^{c}$ & $5.2(4.9,5.6)$ & $5.4(5.0,5.9)$ & $5.6(5.1,6.6)$ & $<.0001$ \\
\hline $\mathrm{TG}, \mathrm{mmol} / \mathrm{L}$ & $1.0(0.7,1.4)$ & $1.3(1.0,1.7)$ & $1.5(1.1,2.1)$ & $1.7(1.2,2.3)$ & $<.0001$ \\
\hline $\mathrm{LDL}-\mathrm{C}, \mathrm{mmol} / \mathrm{L}$ & $2.5 \pm 0.5$ & $2.7 \pm 0.5$ & $2.7 \pm 0.5$ & $2.8 \pm 0.6$ & $<.0001$ \\
\hline $\mathrm{HDL}-\mathrm{C}, \mathrm{mmol} / \mathrm{L}$ & $1.4 \pm 0.3$ & $1.3 \pm 0.2$ & $1.3 \pm 0.2$ & $1.3 \pm 0.2$ & $<.0001$ \\
\hline Uric acid, $\mu \mathrm{mol} / \mathrm{L}$ & $355.6 \pm 68.8$ & $363.6 \pm 75.2$ & $366.0 \pm 74.4$ & $378.0 \pm 77.3$ & $<.0001$ \\
\hline \multicolumn{6}{|l|}{ Women } \\
\hline No. of subjects & 481 & 482 & 480 & 481 & \\
\hline $\mathrm{BMI}, \mathrm{kg} / \mathrm{m}^{2}$ & $20.0 \pm 1.8$ & $22.0 \pm 2.1$ & $23.9 \pm 2.2$ & $26.6 \pm 3.1$ & $<.0001$ \\
\hline $\mathrm{FPG}, \mathrm{mmol} / \mathrm{L}$ & $4.9(4.7,5.2)$ & $5.1(4.8,5.4)$ & $5.1(4.9,5.5)$ & $5.4(5.0,6.0)$ & $<.0001$ \\
\hline $\mathrm{TG}, \mathrm{mmol} / \mathrm{L}$ & $0.8(0.6,1.0)$ & $0.9(0.7,1.3)$ & $1.1(0.8,1.5)$ & $1.4(1.0,1.9)$ & $<.0001$ \\
\hline $\mathrm{LDL}-\mathrm{C}, \mathrm{mmol} / \mathrm{L}$ & $2.5 \pm 0.5$ & $2.6 \pm 0.5$ & $2.8 \pm 0.6$ & $3.0 \pm 0.7$ & $<.0001$ \\
\hline $\mathrm{HDL}-\mathrm{C}, \mathrm{mmol} / \mathrm{L}$ & $1.7 \pm 0.3$ & $1.6 \pm 0.3$ & $1.5 \pm 0.3$ & $1.5 \pm 0.3$ & $<.0001$ \\
\hline Uric acid, $\mu \mathrm{mol} / \mathrm{L}$ & $248.8 \pm 47.1$ & $263.4 \pm 52.1$ & $276.1 \pm 58.7$ & $301.3 \pm 64.8$ & $<.0001$ \\
\hline
\end{tabular}

$B M I$ body mass index, SBP systolic blood pressure, DBP diastolic blood pressure, FPG fasting plasma glucose, $L M M$ leg muscle mass, TC total cholesterol, TG triglycerides, LDL-C low-density lipoprotein cholesterol, HDL-C high-density lipoprotein cholesterol, VFA visceral fat area

Continuous variables are expressed as means \pm standard deviation (SD) and categorical variables are expressed as percentages unless otherwise indicated ${ }^{a} P$ for liner trends are calculated by analysis of variance (for continuous variables) or Cochran-Armitage (for categorical variables)

${ }^{b}$ Data are expressed as medians (interquartile range) 
Table 3 Odds ratio with 95\% confidence interval for the association between VFA-to-LMM ratio and hyperuricemia according to sex

\begin{tabular}{lllll}
\hline Quartiles of VFA-to-LMM ratio & Model 1 & Model 2 & Model 3 & Model 4 \\
\hline Male & & & & \\
Quartile 1 & $1.00($ ref $)$ & $1.00($ ref $)$ & $1.00($ ref $)$ & $1.00($ ref $)$ \\
Quartile 2 & $1.25(0.86,1.82)$ & $1.21(0.83,1.77)$ & $0.91(0.60,1.36)$ & $0.75(0.49,1.15)$ \\
Quartile 3 & $1.58(1.09,2.31)$ & $1.51(1.03,2.21)$ & $1.03(0.67,1.57)$ & $0.79(0.50,1.25)$ \\
Quartile 4 & $2.73(1.89,3.95)$ & $2.49(1.70,3.65)$ & $1.60(1.03,2.49)$ & $0.97(0.55,1.70)$ \\
P for trend & $<.0001$ & $<.0001$ & 0.0180 & 0.9563 \\
Female & & & & $1.00($ ref $)$ \\
Quartile 1 & $1.00($ ref $)$ & $1.00($ ref $)$ & $2.13(0.96,5.21)$ & $1.00($ ref $)$ \\
Quartile 2 & $2.66(1.23,6.42)$ & $2.58(1.19,6.23)$ & $3.74(1.75,8.95)$ & $1.68(0.75,4.17)$ \\
Quartile 3 & $5.20(2.55,12.10)$ & $4.95(2.41,11.54)$ & $5.22(2.44,12.56)$ & $2.42(1.09,6.01)$ \\
Quartile 4 & $8.25(4.05,19.14)$ & $7.37(3.59,17.24)$ & $<.0001$ & $2.57(1.06,6.77)$ \\
P for trend & $<.0001$ & $<.0001$ & 0.0454 \\
\hline
\end{tabular}

Values are ORs ( $95 \% \mathrm{Cls})$ unless otherwise indicated. VFA, visceral fat area; LMM, leg muscle mass.

${ }^{a}$ Model 1: adjusted for age; model 2: adjusted for age, smoking status (for men), menopause status (for women), drinking status, exercise frequency, and history of diseases (hypertension and diabetes); model 3: additionally adjusted for systolic blood pressure, diastolic blood pressure, alanine aminotransferase, fasting plasma glucose, total cholesterol, triglycerides, low-density lipoprotein and high-density lipoprotein cholesterol, and creatinine; model 4: adjusted for model 3 + BMI

${ }^{\mathrm{b}} P$ values for linear trends were calculated using the median value of quartiles of VFA-to-LMM ratio

as the reference), and investigated the risk of hyperuricemia in each group after adjustment by age and gender (Fig. 4). The figure showed that the risk of hyperuricemia rose with the increasing VFA regardless of LMM, while the risk of hyperuricemia increased significantly when the LMM was in the third and highest quantiles. Remarkably, a U-shaped association was found between the risk of hyperuricemia and leg muscle mass, when VFA was in the highest quartile range, and the highest risk of hyperuricemia was discovered when VFA and LMM were both in the highest quantile (OR: 11.50; 95\% CI: 4.86-31.98). The results suggested that there was an interaction between VFA and LMM, and the rising of VFA and LMM would lead to a rapid increase of hyperuricemia risks.

\section{Discussion}

In the present study, we explored the association of the VFA-to-LMM ratio with hyperuricemia. The results showed that the VFA-to-LMM ratio was positively associated with the risk of hyperuricemia in women after adjustment by age, BMI, smoking status (for males), menopause status (for females), drinking status, exercise frequency, SBP, DBP, ALT, FPG, TC, TG, LDL-C, HDL$\mathrm{C}$, creatinine, and history of diseases. In addition, the highest risk of hyperuricemia was discovered when both VFA and LMM were at the highest quartile.

At present, the association between visceral fat mass and hyperuricemia has been confirmed by several studies. A study conducted by Huang et al. [26] found that visceral adipose accumulation was closely associated with hyperuricemia in Chinese adults. Similarly, a study from Takahashi et al. [27] confirmed the important role of visceral fat accumulation in increased serum uric acid levels in obese men. Both studies confirmed a significant association between increased visceral fat mass and hyperuricemia, and further compared the contribution of visceral and subcutaneous fat to the association. Consistent with previous studies, our results also showed a significant positive association between VFA and
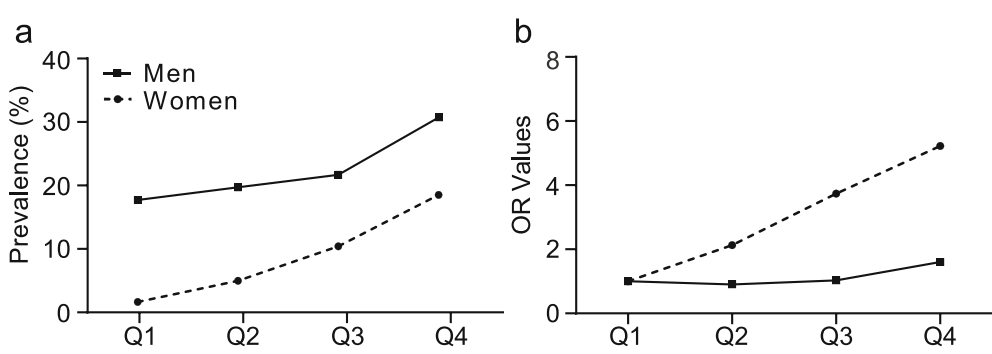

Quartiles of VFA-to-LMM ratio

Fig. 3 The prevalence (a) and odds ratio (b) for hyperuricemia according to quartiles of the VFA-to-LMM ratio between men and women 
Table 4 Subgroup analysis of the association between VFA-to-LMM ratio and hyperuricemia according to menopausal status

\begin{tabular}{|c|c|c|c|c|}
\hline Menopausal status & Model $1^{a}$ & Model 2 & Model 3 & Model 4 \\
\hline \multicolumn{5}{|l|}{ Pre-menopausal } \\
\hline Quartile 1 & 1.00 (ref) & 1.00 (ref) & 1.00 (ref) & 1.00 (ref) \\
\hline Quartile 2 & $3.50(1.06,15.75)$ & $3.65(1.10,16.47)$ & $3.63(1.04,17.10)$ & $2.78(0.79,13.06)$ \\
\hline Quartile 3 & $5.01(1.61,21.95)$ & $5.30(1.70,23.27)$ & $4.62(1.36,21.65)$ & $2.84(0.78,13.74)$ \\
\hline Quartile 4 & $12.04(4.21,50.78)$ & $12.97(4.50,54.99)$ & $8.25(2.52,37.99)$ & $3.61(0.90,18.86)$ \\
\hline$P$ for trend ${ }^{b}$ & $<.0001$ & $<.0001$ & 0.0005 & 0.1503 \\
\hline \multicolumn{5}{|l|}{ Post-menopausal } \\
\hline Quartile 1 & 1.00 (ref) & 1.00 (ref) & 1.00 (ref) & 1.00 (ref) \\
\hline Quartile 2 & $2.52(1.29,5.19)$ & $2.50(1.27,5.18)$ & $2.18(1.05,4.73)$ & $1.79(0.84,3.96)$ \\
\hline Quartile 3 & $2.36(1.20,4.89)$ & $2.25(1.14,4.69)$ & $1.85(0.88,4.03)$ & $1.35(0.61,3.09)$ \\
\hline Quartile 4 & $3.71(1.95,7.51)$ & $3.43(1.79,6.99)$ & $3.33(1.64,7.13)$ & $1.94(0.80,4.81)$ \\
\hline$P$ for trend & 0.0003 & 0.0010 & 0.0029 & 0.3130 \\
\hline
\end{tabular}

Values are ORs (95\% Cls) unless otherwise indicated. VFA visceral fat area, LMM leg muscle mass

${ }^{a}$ Model 1: adjusted for age; model 2: adjusted for age, drinking status, exercise frequency, and history of diseases (hypertension and diabetes); model 3 :

additionally adjusted for systolic blood pressure, diastolic blood pressure, alanine aminotransferase, fasting plasma glucose, total cholesterol, triglycerides, lowdensity lipoprotein and high-density lipoprotein cholesterol, and creatinine. model 4: adjusted for model $3+\mathrm{BMI}$

${ }^{\mathrm{b}} \mathrm{P}$ values for linear trends were calculated using the median value of quartiles of VFA-to-LMM ratio

hyperuricemia. However, there were still limited studies on the association between skeletal muscle mass of lower limbs and hyperuricemia. In the cross-sectional study based on 7544 adults, it revealed that serum uric acid was negatively associated with skeletal muscle mass index [15]. Similarly, Tanaka et al. [28] found that a higher SUA level was associated with reduced muscle mass in men with diabetes. Conversely, several studies unveiled that a high serum uric acid level was a protective factor of muscle function [2931 , which could counteract the excessive production of free radicals that cause muscle protein damages and eventually lead to the decline of muscle mass and strength [32].

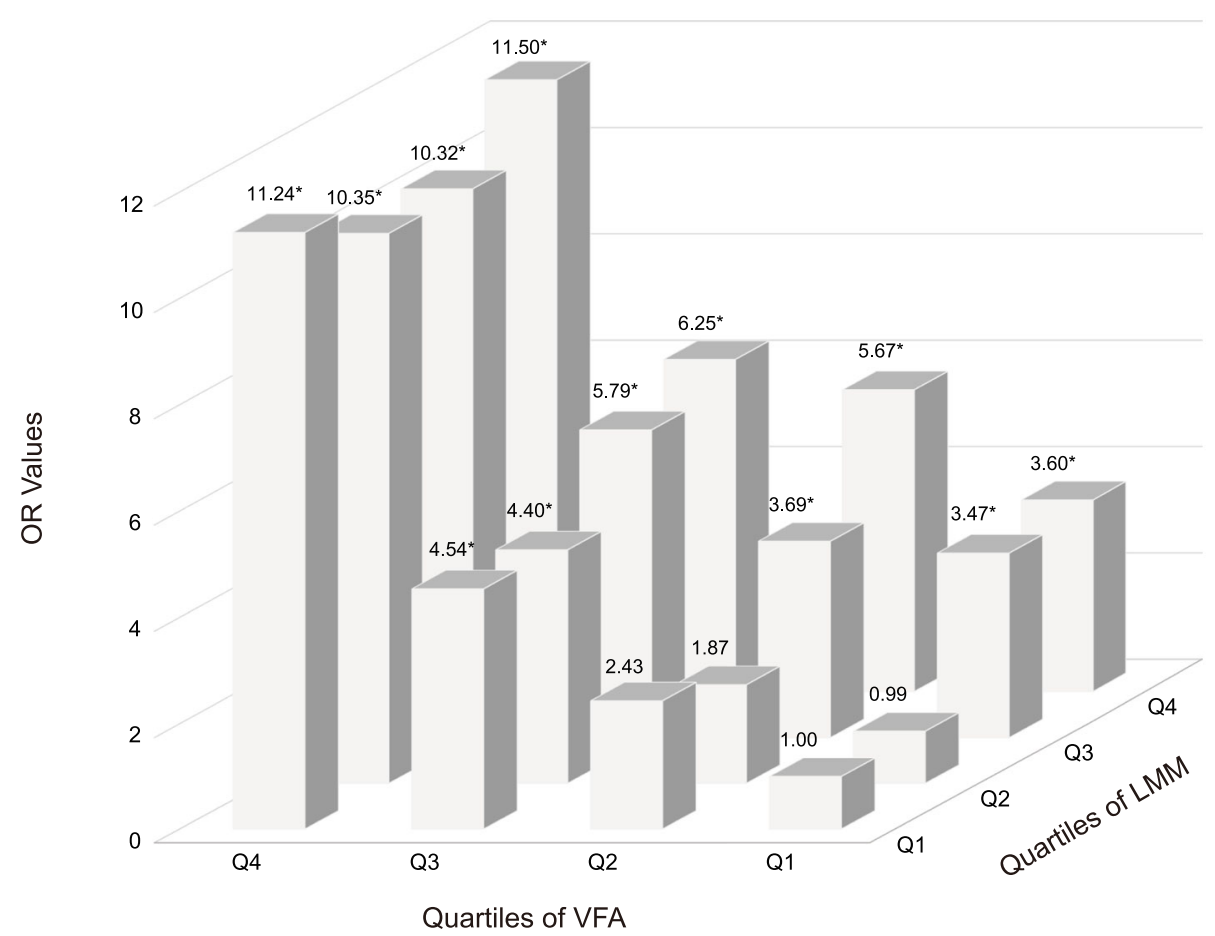

Fig. 4 Age- and gender-adjusted odds ratios for hyperuricemia according to the quartiles of VFA and LMM. *The $95 \%$ confidence interval does not contain 1 
In the current study, it is found that the increase of skeletal muscle mass (or the VFA-to-SMM ratio) was not associated with hyperuricemia after adjustment by BMI, but the VFA-to-LMM ratio was independently associated with the risk of hyperuricemia. The results indicated that the relative increase in skeletal muscle mass of the lower limbs has made a great contribution to the formation of hyperuricemia. There are several indirect evidences that provide partial support. For example, in a previous study, it was found that thigh circumference, a comprehensive indicator of leg muscle mass, was strongly associated with diabetes and could be used as a good predictor [33]. In another study conducted by Min et al., it was found that there was a negative association between thigh circumference and peripheral arterial diseases when thigh circumference was less than $55 \mathrm{~cm}$ [34]. The preceding evidence showed that the changes of leg composition are closely related to body metabolism and health status, which may promote the formation of hyperuricemia.

The underlying mechanism of the association between body composition and hyperuricemia has not been clearly elucidated. The results might be analyzed in the following three aspects: Firstly, with the accumulation of visceral fat, some pro-inflammatory cytokines, such as IL-6, IL-8, monocyte chemoattractant protein 1 (MPC1 ), and tumor necrosis factor $\alpha$ (TNF- $\alpha$ ), secreted or induced by adipose tissue can lead to low-grade inflammation and oxidative stress, and further cause insulin resistance. In addition, non-esterified fatty acids (NEEAs) and resistin secreted by adipose tissue were directly related to insulin resistance $[35,36]$. As the prime target of insulin, skeletal muscle was extremely important for maintaining the homeostasis of glucose and fatty acid metabolism in body. The decrease of skeletal muscle (especially leg muscle) greatly affected the metabolism of plasma fatty acid and glucose, which was further associated with insulin resistance [37]. Obviously, both visceral fat and skeletal muscle were closely associated with insulin resistance, which can lead to hyperuricemia in the following three ways: (1) IR can directly affect the reabsorption of uric acid by renal tubules and ultimately lead to the formation of hyperuricemia [38, 39]; (2) IR can indirectly cause hyperinsulinemia, which in turn provokes hyperuricemia; and (3) IR could indirectly increase the production of NADPH by promoting the lipolysis pathway, which is an important source of serum uric acid and eventually leads to hyperuricemia $[40,41]$. The results showed that the increase of the VFA-to-LMM ratio led to a higher risk of hyperuricemia than the increase of VFA alone, indicating that increased LMM may play a protective role in the association, which was consistent with previous studies. Secondly, as far as we know, visceral fat accumulation is significantly associated with metabolic syndrome, while relative skeletal muscle mass was confirmed to be inversely associated with the development of metabolic syndrome [42]. Similarly, some studies have confirmed that SUA was closely related to the metabolic syndrome and may have a dual effect of cause and effect $[6,43]$. It is worth noting that Kim et al. also confirmed that women with metabolic syndrome had a higher risk of hyperuricemia when they were compared with men, which was consistent with our results. Besides, some studies have shown that glucose and lipid metabolic disorder is related to the abnormal secretion of leptin, which induces oxidative stress in endothelial cells, and further leads to the increase of serum uric acid $[44,45]$. Some studies have also shown that leptin, as a regulator of serum uric acid concentration, may be an intermediate factor between obesity and hyperuricemia [46]. Finally, our results showed significant gender differences, which were significant after adjustment by BMI. As we all know, there are considerable differences in both the distribution and mass of body fat and skeletal muscle between men and women due to the differences of gender hormone levels $[47,48]$. Compared with women, men have a higher VFA and relatively lower SMM (or LMM), which makes the VFA-to-LMM ratio much lower than that of women. This may explain why the prevalence of hyperuricemia in men is higher than that in women, but the risk of hyperuricemia in women becomes higher as the VFA-toLMM ratio increases. In addition, after adjustment by BMI, the association between the VFA-to-LMM ratio and hyperuricemia disappeared in men, suggesting a linear correlation between the VFA-to-LMM ratio and BMI, but for women, it is different. Therefore, the VFAto-LMM ratio seems to have more clinical values in predicting the risk of hyperuricemia in women.

There are three advantages in the present study. First of all, as far as we know, this is the first study on the association between the VFA-to-LMM ratio and hyperuricemia. A comprehensive analysis was conducted on body composition, including VFA, LMM, and their interactions. Secondly, all measurements and statistical analysis were performed in strict accordance with the standard procedures to ensure data accuracy. Thirdly, given the significant gender differences in body composition, men and women were stratified in the analysis to ensure the reliability of the results. However, several limitations also need to be mentioned. Firstly, based on the cross-sectional data, the causality of the VFA-to-LMM ratio and hyperuricemia could not be obtained, and the potential mechanism could not be further analyzed. Secondly, although confounding factors had been adjusted as many as possible, there were still factors that were not included. However, many confounders were adjusted as possible to ensure the robustness of the results. 
Thirdly, due to the limitation of the sample size, further study is still needed to verify whether the association between the VFA-to-LMM ratio and hyperuricemia in subgroup analysis of women is independent of BMI.

\section{Perspectives and significance}

According to the results, the metabolism of uric acid can be indirectly reflected in body shape, and the indicator studied in this paper can well predict the risk of hyperuricemia in women. The findings facilitated the prediction of high-risk groups of hyperuricemia by simple body composition examination or even apparent body shape. The results of subgroup analysis in the female population suggested that more attention should be paid to the changes of the VFA and LMM in premenopausal women. In addition, the results provided potential values for the prevention of hyperuricemia.

\section{Conclusion}

According to the research results, the VFA-to-LMM ratio is positively associated with the risk of hyperuricemia in women after adjustment by confounding factors, and women are more sensitive than men to the risk of hyperuricemia caused by changes in body composition. In addition, pre-menopausal women have a higher risk to suffer from hyperuricemia than post-menopausal women with the increase of the VFA-to-LMM ratio. However, well-controlled prospective studies are needed to further confirm the causality between the VFA-to-LMM ratio and hyperuricemia.

\section{Abbreviations \\ ALT: Alanine aminotransferase; BMI: Body mass index; Cl: Confidence interval: DBP: Diastolic blood pressure; FPG: Fasting plasma glucose; HDL-C: High- density lipoprotein cholesterol; IR: Insulin resistance; LDL-C: Low-density lipoprotein cholesterol; LMM: Leg muscle mass; NAFLD: Non-alcoholic fatty liver disease; OR: Odds ratio; SMM: Skeletal muscle mass; SUA: Serum uric acid; SBP: Systolic blood pressure; TC: Total cholesterol; TG: Triglycerides; VFA: Visceral fat area}

\section{Acknowledgements}

We gratefully acknowledge all the participants of the study, Tianjin Union Medical Center-Health Management Department and Nankai University Affiliated Hospital and for the possibility to perform the study. All data generated during this study can be freely obtained with the permission of corresponding authors.

\section{Authors' contributions \\ $\mathrm{CJL}$ and JNL contributed to the concept and design of the study; WRJ and XHW contributed to the design of analysis, statistical analysis, and manuscript preparation; CJL, WRJ, XHW, YPJ, MYZ, and YXS contributed to coordination of the fieldwork, data collection and management, and interpretation of results and revision of manuscript drafts. All authors were involved in the interpretation of the results and the revision of the manuscript and approved the submitted version of the manuscript.}

\section{Funding}

This work was partially supported by Grants from Natural Science Foundation of Tianjin (19JCZDJC36100 to C.J.L and 18ZXDBSY22120 to J.N.L) and National Key R\&D Program of China (2016YFC0900600, 2016YFC0900604 to M.Y.Z).

\section{Availability of data and materials}

The datasets used and analyzed during the current study are available from the corresponding author on reasonable request.

\section{Ethics approval and consent to participate}

This study was approved by the Institutional Review Board of Tianjin Union Medical Center, Nankai University affiliated hospital. All participants were informed about the study objectives and examination procedures in detail, and were asked to sign the informed consent form before they were enrolled.

\section{Consent for publication}

Not applicable.

\section{Competing interests}

All authors have no conflicts of interest to disclose.

\section{Author details}

${ }^{1}$ Department of Endocrinology, Health Management Center, Tianjin Union Medical Center, Nankai University Affiliated Hospital, 190 of Jieyuan Road, Hongqiao District, Tianjin 300121, China. ${ }^{2}$ College of Medicine, Nankai University, Tianjin, China. ${ }^{3}$ Orofacial Pain and TMJ Disorders, Eastman Institute for Oral Health, University of Rochester, New York, NY, USA.

Received: 29 September 2020 Accepted: 17 January 2021

Published online: 29 January 2021

\section{References}

1. Chen-Xu M, Yokose C, Rai SK, Pillinger MH, Choi HK. Contemporary prevalence of gout and hyperuricemia in the United States and decadal trends: the national health and nutrition examination survey, 2007-2016. Arthritis Rheum. 2019;71(6):991-9. https://doi.org/10.1002/art.40807.

2. Liu R, Han C, Wu D, Xia X, Gu J, Guan H, et al. Prevalence of hyperuricemia and gout in mainland China from 2000 to 2014: a systematic review and meta-analysis. Biomed Res Int. 2015;2015:762820. https://doi.org/10.1155/ 2015/762820

3. Yang WX, Ma Y, Hou YL, Wang YB, You CG. Prevalence of hyperuricemia and its correlation with serum lipids and blood glucose in physical examination population in 2015-2018: a retrospective study. Clin Lab. 2019; 65(8):1. https://doi.org/10.7754/Clin.Lab.2019.190338.

4. Simao AN, Lozovoy MA, Dichi I. The uric acid metabolism pathway as a therapeutic target in hyperuricemia related to metabolic syndrome. Expert Opin Ther Targets. 2012;16(12):1175-87. https://doi.org/10.1517/14728222. 2012.723694.

5. Yu TY, Jee JH, Bae JC, Jin SM, Baek JH, Lee MK, et al. Serum uric acid: a strong and independent predictor of metabolic syndrome after adjusting for body composition. Metabolism. 2016;65(4):432-40. https://doi.org/10. 1016/j.metabol.2015.11.003.

6. Hayden MR, Tyagi SC. Uric acid: a new look at an old risk marker for cardiovascular disease, metabolic syndrome, and type 2 diabetes mellitus: the urate redox shuttle. Nutr Metab. 2004;1(1):10. https://doi.org/10.1186/ 1743-7075-1-10.

7. Kuwabara M, Kuwabara R, Hisatome I, Niwa K, Roncal-Jimenez CA, Bjornstad $P$, et al. "Metabolically healthy" obesity and hyperuricemia increase risk for hypertension and diabetes: 5-year Japanese cohort study. Obesity (Silver Spring). 2017;25(11):1997-2008. https://doi.org/10.1002/oby.22000.

8. Sandra S, Lesmana CRA, Purnamasari D, Kurniawan J, Gani RA Hyperuricemia as an independent risk factor for non-alcoholic fatty liver disease (NAFLD) progression evaluated using controlled attenuation parameter-transient elastography: lesson learnt from tertiary referral center. Diabetol Metab Syndr. 2019;13(1):424-8. https://doi.org/10.1016/.jdsx.2018. 10.001

9. Xie Y, Xu P, Liu K, Lin S, Wang M, Tian T, et al. Hyperuricemia and gout are associated with cancer incidence and mortality: a meta-analysis based on cohort studies. J Cell Physiol. 2019;234(8):14364-76. https://doi.org/10.1002/ jcp.28138.

10. Choi HK, McCormick N, Lu N, Rai SK, Yokose C, Zhang Y. Population impact attributable to modifiable risk factors for hyperuricemia. Arthritis Rheum. 2020;72(1):157-65. https://doi.org/10.1002/art.41067.

11. Han T, Meng X, Shan R, Zi T, Li Y, Ma H, et al. Temporal relationship between hyperuricemia and obesity, and its association with future risk of 
type 2 diabetes. Int J Obes. 2018;42(7):1336-44. https://doi.org/10.1038/ s41366-018-0074-5.

12. Yamada A, Sato KK, Kinuhata S, Uehara S, Endo G, Hikita Y, et al. Association of Visceral fat and Liver fat with Hyperuricemia. Arthritis Care Res. 2016; 68(4):553-61. https://doi.org/10.1002/acr.22729.

13. Matsuura F, Yamashita S, Nakamura T, Nishida M, Nozaki S, Funahashi T, et al. Effect of visceral fat accumulation on uric acid metabolism in male obese subjects: visceral fat obesity is linked more closely to overproduction of uric acid than subcutaneous fat obesity. Metabolism. 1998;47(8):929-33. https://doi.org/10.1016/s0026-0495(98)90346-8.

14. Dong $H, X u Y$, Zhang $X$, Tian S. Visceral adiposity index is strongly associated with hyperuricemia independently of metabolic health and obesity phenotypes. Sci Rep. 2017;7(1):8822. https://doi.org/10.1038/s41598017-09455-z.

15. Beavers KM, Beavers DP, Serra MC, Bowden RG, Wilson RL. Low relative skeletal muscle mass indicative of sarcopenia is associated with elevations in serum uric acid levels: findings from NHANES III. J Nutr Health Aging. 2009;13(3):177-82. https://doi.org/10.1007/s12603-009-0054-5.

16. Alvim RO, Siqueira JH, Zaniqueli D, Dutra DM, Oliosa PR, Mill JG. Influence of muscle mass on the serum uric acid levels in children and adolescents. Nutr Metab Cardiovasc Dis. 2020;30(2):300-5. https://doi.org/10.1016/j.numecd. 2019.08.019

17. Heshka S, Ruggiero A, Bray GA, Foreyt J, Kahn SE, Lewis CE, et al. Altered body composition in type 2 diabetes mellitus. Int J Obes. 2008;32(5):780-7. https://doi.org/10.1038/sj.ijo.0803802.

18. Jun DW, Han JH, Kim SH, Jang EC, Kim NI, Lee JS, et al. Association between low thigh fat and non-alcoholic fatty liver disease. J Gastroenterol Hepatol. 2008;23(6):888-93. https://doi.org/10.1111/j.1440-1746.2008.05330.x.

19. Park JS, Cho MH, Ahn CW, Kim KR, Huh KB. The association of insulin resistance and carotid atherosclerosis with thigh and calf circumference in patients with type 2 diabetes. Cardiovasc Diabetol. 2012;11:62. https://doi. org/10.1186/1475-2840-11-62

20. Snijder MB, Dekker JM, Visser M, Bouter LM, Stehouwer CD, Kostense PJ, et al. Associations of hip and thigh circumferences independent of waist circumference with the incidence of type 2 diabetes: the Hoorn study. Am J Clin Nutr. 2003:77(5):1192-7. https://doi.org/10.1093/ajcn/77.5.1192.

21. Snijder MB, Visser M, Dekker JM, Goodpaster BH, Harris TB, Kritchevsky SB, et al. Low subcutaneous thigh fat is a risk factor for unfavourable glucose and lipid levels, independently of high abdominal fat. The health $A B C$ study. Diabetologia. 2005;48(2):301-8. https://doi.org/10.1007/s00125-004-1637-7.

22. Lima WG, Martins-Santos ME, Chaves VE. Uric acid as a modulator of glucose and lipid metabolism. Biochimie. 2015;116:17-23. https://doi.org/10. 1016/j.biochi.2015.06.025

23. Jiao X, Zhang G, Li G, Yin X, Shen X. Analysis on correlations of serum uric acid with glucose and lipid metabolism and renal function of patients with newly-diagnosed and long-term type 2 diabetes mellitus. Panminerva Med. 2019Epub ahead of print. https://doi.org/10.23736/S0031-0808.19.03635-8.

24. Farrell GC, Chitturi S, Lau GK, Sollano JD. Guidelines for the assessment and management of non-alcoholic fatty liver disease in the Asia-Pacific region: executive summary. J Gastroenterol Hepatol. 2007;22(6):775-7. https://doi. org/10.1111/j.1440-1746.2007.05002x.x

25. Joint committee for guideline r. 2016 Chinese guidelines for the management of dyslipidemia in adults. J Geriatr Cardiol. 2018;15(1):1-29. https://doi.org/10.11909/j.issn.1671-5411.2018.01.011.

26. Huang $X$, Jiang $X$, Wang $L$, Chen $L$, Wu $Y$, Gao $P$, et al. Visceral adipose accumulation increased the risk of hyperuricemia among middle-aged and elderly adults: a population-based study. J Transl Med. 2019;17(1):341. https://doi.org/10.1186/s12967-019-2074-1

27. Takahashi S, Yamamoto T, Tsutsumi Z, Moriwaki Y, Yamakita J, Higashino K. Close correlation between visceral fat accumulation and uric acid metabolism in healthy men. Metabolism. 1997;46(10):1162-5. https://doi. org/10.1016/s0026-0495(97)90210-9.

28. Tanaka Kl, Kanazawa I, Notsu M, Sugimoto T. Higher serum uric acid is a risk factor of reduced muscle mass in men with type 2 diabetes mellitus. Exp Clin Endocrinol Diabetes. 2018Epub ahead of print. https://doi.org/10.1055/ a-0805-2197.

29. Molino-Lova R, Sofi F, Pasquini G, Vannetti F, Del Ry S, Vassalle C, et al. Higher uric acid serum levels are associated with better muscle function in the oldest old: results from the Mugello study. Eur J Intern Med. 2017;41:3943. https://doi.org/10.1016/j.ejim.2017.03.014
30. Wu Y, Zhang D, Pang Z, Jiang W, Wang S, Tan Q. Association of serum uric acid level with muscle strength and cognitive function among Chinese aged 50-74 years. Geriatr Gerontol Int. 2013;13(3):672-7. https://doi.org/10. 1111/j.1447-0594.2012.00962.x.

31. Dong XW, Tian HY, He J, Wang C, Qiu R, Chen YM. Elevated serum uric acid is associated with greater bone mineral density and skeletal muscle mass in middle-aged and older adults. PLoS One. 2016;11(5):e0154692. https://doi. org/10.1371/journal.pone.0154692.

32. Macchi C, Molino-Lova R, Polcaro P, Guarducci L, Lauretani F, Cecchi F, et al. Higher circulating levels of uric acid are prospectively associated with better muscle function in older persons. Mech Ageing Dev. 2008;129(9):522-7. https://doi.org/10.1016/j.mad.2008.04.008.

33. Jung KJ, Kimm H, Yun JE, Jee SH. Thigh circumference and diabetes: obesity as a potential effect modifier. J Epidemiol. 2013;23(5):329-36. https://doi. org/10.2188/jea.je20120174.

34. Min JY, Cho JS, Lee KJ, Park JB, Min KB. Thigh circumference and low ankle brachial index in US adults: results from the National Health and nutrition examination survey 1999-2004. Int J Cardiol. 2013;163(1):40-5. https://doi. org/10.1016/j.ijcard.2012.10.039.

35. Frayn KN. Adipose tissue as a buffer for daily lipid flux. Diabetologia. 2002; 45(9):1201-10. https://doi.org/10.1007/s00125-002-0873-y.

36. Gustafson B. Adipose tissue, inflammation and atherosclerosis. J Atheroscler Thromb. 2010;17(4):332-41. https://doi.org/10.5551/jat.3939.

37. Sacchetti M, Olsen DB, Saltin B, van Hall G. Heterogeneity in limb fatty acid kinetics in type 2 diabetes. Diabetologia. 2005;48(5):938-45. https://doi.org/ 10.1007/s00125-005-1727-1.

38. Facchini F, Chen YD, Hollenbeck CB, Reaven GM. Relationship between resistance to insulin-mediated glucose uptake, urinary uric acid clearance, and plasma uric acid concentration. JAMA. 1991;266(21):3008-11. https:// doi.org/10.1001/jama.1991.03470210076036.

39. Choi HK, Mount DB, Reginato AM. American College of $P$, American physiological S. pathogenesis of gout. Ann Intern Med. 2005;143(7):499-516. https://doi.org/10.7326/0003-4819-143-7-200510040-00009.

40. Clausen JO, Borch-Johnsen K, Ibsen H, Pedersen O. Analysis of the relationship between fasting serum uric acid and the insulin sensitivity index in a population-based sample of 380 young healthy Caucasians. Eur J Endocrinol. 1998;138(1):63-9. https://doi.org/10.1530/eje.0.1380063.

41. Vuorinen-Markkola H, Yki-Jarvinen $\mathrm{H}$. Hyperuricemia and insulin resistance. J Clin Endocrinol Metab. 1994;78(1):25-9. https://doi.org/10.1210/jcem.78.1. 8288709 .

42. Kim G, Lee SE, Jun JE, Lee YB, Ahn J, Bae JC, et al. Increase in relative skeletal muscle mass over time and its inverse association with metabolic syndrome development: a 7-year retrospective cohort study. Cardiovasc Diabetol. 2018;17(1):23. https://doi.org/10.1186/s12933-018-0659-2.

43. Kim IY, Han KD, Kim DH, Eun Y, Cha HS, Koh EM, et al. Women with metabolic syndrome and general obesity are at a higher risk for significant hyperuricemia compared to men. J Clin Med. 2019;8(6):837. https://doi.org/ 10.3390/jcm8060837.

44. D'Elia L, Giaquinto A, Cappuccio FP, lacone R, Russo O, Strazzullo P, et al. Circulating leptin is associated with serum uric acid level and its tubular reabsorption in a sample of adult middle-aged men. J Endocrinol Investig. 2020;43(5):587-93. https://doi.org/10.1007/s40618-019-01140-4.

45. Lin JD, Chiou WK, Chang HY, Liu FH, Weng HF. Serum uric acid and leptin levels in metabolic syndrome: a quandary over the role of uric acid. Metabolism. 2007;56(6):751-6. https://doi.org/10.1016/..metabol.2007.01.006.

46. Bedir A, Topbas M, Tanyeri F, Alvur M, Arik N. Leptin might be a regulator of serum uric acid concentrations in humans. Japanese Heart J. 2003:44(4): 527-36. https://doi.org/10.1536/jhj.44.527.

47. Shimokata H, Tobin JD, Muller DC, Elahi D, Coon PJ, Andres R. Studies in the distribution of body fat: I. effects of age, sex, and obesity. J Gerontol. 1989; 44(2):M66-73. https://doi.org/10.1093/geronj/44.2.m66.

48. Karastergiou K, Smith SR, Greenberg AS, Fried SK. Sex differences in human adipose tissues_the biology of pear shape. Biol Sex Differ. 2012;3(1):13. https://doi.org/10.1186/2042-6410-3-13.

\section{Publisher's Note}

Springer Nature remains neutral with regard to jurisdictional claims in published maps and institutional affiliations. 